\title{
Aqueous extracts of Sanghuangporus vaninii induce S-phase arrest and apoptosis in human melanoma A375 cells
}

\author{
TAIHEN YU ${ }^{1}$, SHI ZHONG ${ }^{2}$, YUQING SUN ${ }^{2}$, HAIYAN SUN ${ }^{3}$, WEIGUO CHEN ${ }^{3}$, \\ YOUGUI LI ${ }^{2}$, JIANXUN ZHU ${ }^{2}$, LONGXI LU ${ }^{4}$ and JINXI HUO ${ }^{2}$

\begin{abstract}
${ }^{1}$ Department of Radiology, Zhejiang Provincial People's Hospital, Affiliated People's Hospital of Hangzhou Medical College, Hangzhou, Zhejiang 310014; ${ }^{2}$ Institute of Sericultural and Tea, Zhejiang Academy of Agricultural Sciences, Hangzhou, Zhejiang 310021; ${ }^{3}$ Haining Economic Crop Technical Service Station, Haining Agricultural and Rural Bureau, Haining, Zhejiang 314400; ${ }^{4}$ Department of Environment \& Health, Zhejiang Provincial Center for Disease Control and Prevention,
\end{abstract} \\ Hangzhou, Zhejiang 310051, P.R. China
}

Received April 8, 2021; Accepted June 15, 2021

DOI: $10.3892 / \mathrm{ol} .2021 .12889$

\begin{abstract}
Sanghuangporus vaninii, also called 'Sanghuang' mushroom in Chinese, has various medicinal uses, but its effects on human melanoma cells have not been reported. The present study investigated the inhibitory ability and potential anticancer mechanism of the aqueous extracts of S. vaninii $(\mathrm{SH})$. The results revealed that $\mathrm{SH}$ inhibited the proliferation of A375 human melanoma cells in a dose-dependent manner, and flow cytometry analysis suggested that SH induced A375 cell cycle arrest at S phase and apoptosis. Reverse transcription-quantitative PCR, western blotting and immunofluorescence analyses indicated that $\mathrm{SH}$ induced $\mathrm{S}$-phase arrest by upregulating p21 expression, and p21 inhibited the expression of cyclin-cyclin-dependent kinases complexes at both the RNA and protein levels. In addition, $\mathrm{SH}$ induced apoptosis of A375 cells by inhibiting the expression levels of the anti-apoptosis gene $\mathrm{Bcl}-2$. Therefore, the results suggested that $\mathrm{SH}$ may be a potential candidate for the treatment of human melanoma, thus providing new ideas for developing drugs that target melanoma.
\end{abstract}

\section{Introduction}

Melanoma is one of the most aggressive types of cancer with an unpredictable course of disease. It accounts for $4 \%$ of all skin

Correspondence to: Dr Longxi Lu, Department of Environment \& Health, Zhejiang Provincial Center for Disease Control and Prevention, 3399 Binsheng Road, Hangzhou, Zhejiang 310051, P.R. China

E-mail:1xilu@cdc.zj.cn

Dr Jinxi Huo, Institute of Sericultural and Tea, Zhejiang Academy of Agricultural Sciences, 298 Desheng Road, Hangzhou, Zhejiang 310021, P.R. China

E-mail: huojx@zaas.ac.cn

Key words: Sanghuangporus vaninii, aqueous extracts, S-phase arrest, apoptosis, human melanoma cancer cases but is responsible for $75 \%$ of deaths caused by these malignancies (1). Surgical removal is the best treatment for localised melanoma. For patients with metastatic disease, surgical treatment alone will not be curative, and chemotherapy and targeted therapy are necessary (2). However, the prognosis for patients treated by chemotherapy is unsatisfactory, and the side effects are serious, and the targeted therapy is limited to specific genotype patients (2). Therefore, effective drugs should be developed for the treatment of melanoma.

Sanghuangporus spp., also called 'Sanghuang' in China, are a well-known medicinal mushroom (3). Sanghuang have been used in Chinese traditional medicine for $>2,000$ years for the treatment of various diseases such as haemorrhage, haemostasis and diseases related to female menstruation (4). In recent years, modern pharmacological researches have revealed that Sanghuangporus spp. have multiple functions, such as anti-carcinogenesis, anti-inflammatory, anti-oxidative, anti-fungal and immunomodulatory activities $(5,6)$. The extracts of $S$. rimosus have antiproliferative and apoptotic activities on human colorectal carcinoma HCT116 cells (7). The total ethanol extracts of $S$. igniarius have antitumor activities against gastric cancer SGC-7901 cells through a mitochondria-dependent pathway (8). Im et al (9) found that the extracts of $S$. vaninii are cytotoxic to murine melanoma B16-F10 cells at $25-750 \mathrm{mg} / \mathrm{ml}$. Bae et al $(10,11)$ demonstrated that polysaccharides from $S$. gilvus can inhibit forestomach carcinogenesis in mice and the proliferation of murine melanoma cells. Protocatechualdehyde induces S-phase arrest and apoptosis by stimulating the $\mathrm{p} 27^{\mathrm{KIP} 1}$-CyclinA/D1-cyclin-dependent kinase 2 (CDK2) and mitochondrial apoptotic pathways in HT-29 cells (12). 3,4-Dihydroxybenzalactone isolated from $S$. linteus suppresses human non-small cell lung carcinoma migration and invasiveness via the ROS-mediated PI3K/AKT/MAPK/MMP and NFKB signalling pathways (13). 3,4-Dihydroxybenzalacetone isolated from $S$. obliguus promotes anti-melanogenesis by downregulating melanosome maturation and transportation in murine melanoma B16F10 cells and human epidermal melanocytes (14). The extracts of $S$. baumii exert antitumour effects on human melanoma A375 cells in vitro and in vivo (15). S. gilvus-derived 
protocatechualdehyde induces $\mathrm{G}_{0} / \mathrm{G}_{1}$-phase arrest and apoptosis in murine B16-F10 cells (16). Therefore, Sanghuang has important inhibitory effects on melanoma. However, the effect of S. vaninii on human melanoma has not been reported. The present study revealed that aqueous extracts of $S$. vaninii fruiting body (referred as $\mathrm{SH}$ ) acted as potential inhibitors of melanoma by inhibiting the proliferation of A375 cells and inducing cell cycle arrest and apoptosis.

\section{Materials and methods}

Cells and kits. The human melanoma A375 cell line was obtained from the Institute of Biochemistry and Cell Biology of the Chinese Academy of Sciences. The cells were cultured in RPMI-1640 medium (Thermo Fisher Scientific, Inc.) at $37^{\circ} \mathrm{C}$ with $5 \% \mathrm{CO}_{2}$. The medium was supplemented with 10\% FBS (Thermo Fisher Scientific,Inc.), penicillin $(100 \mu \mathrm{g} / \mathrm{ml})$ and streptomycin $(100 \mu \mathrm{g} / \mathrm{ml})$. MTT was obtained from Sigma-Aldrich (Merck KGaA). The Dead Cell Apoptosis kit with FITC Annexin V and PI was obtained from Thermo Fisher Scientific, Inc.

Preparation of aqueous extracts of $S$. vaninii fruiting body. The fruiting body of $S$. vaninii was cultivated at the Sericultural Research Institute of Zhejiang Academy of Agricultural Sciences (Hangzhou, China). The dried fruiting body powder was extracted with boiling water for $2 \mathrm{~h}$. The supernatant was collected after centrifugation at $8,000 \mathrm{x} \mathrm{g}$ at room temperature for $10 \mathrm{~min}$. The supernatant was lyophilised to obtain extract powders. The content of total polysaccharides and polyphenols was 5.51 and $23.00 \%$, respectively. The major polyphenols were 3,4-dihydroxybenzalacetone, phellibaumin D and interfungin B (17).

Cell proliferation assay. Cell proliferation was determined by trypan blue staining and the inhibitory ratio was measured by MTT-based colorimetric assay as described by Zhong et al (16). Briefly, cells at the exponential growth phase were dispensed into a 96-well microplate at $100 \mu 1 /$ well. After $24 \mathrm{~h}, 100 \mu \mathrm{l}$ of different concentrations of SH were added to prepare final concentrations of 50, 100, 200 and $400 \mu \mathrm{g} / \mathrm{ml}$. Sterile water $(0.1 \%$ DMSO) was used as control. The cells were cultured at $37^{\circ} \mathrm{C}$ for 24,36 and $48 \mathrm{~h}$ for cell counting. The supernatants of each well were obtained, and $50 \mu 1$ of $0.25 \%$ trypsin was added into each well. After incubation at $37^{\circ} \mathrm{C}$ for $5 \mathrm{~min}$, the supernatants were re-added to the corresponding well to stop cell digestion. Cell suspensions $(\sim 20 \mu \mathrm{l})$ were transferred into a new $1.5 \mathrm{ml}$ centrifuge tube with $20 \mu \mathrm{l}$ of $0.4 \%$ trypan blue solution. After mixing, $20 \mu \mathrm{l}$ cell suspension was added to a cell counting plate for counting with an automatic cell counter (IC1000; Countstar; ALIT Life Science Co., Ltd.). Cells cultured for $48 \mathrm{~h}$ were used for the MTT assay. The cells were incubated at $37^{\circ} \mathrm{C}$ with $50 \mu 1$ of MTT solution $(1 \mathrm{mg} / \mathrm{ml})$ for $2 \mathrm{~h}$, and the resulting crystals were dissolved in DMSO. The absorbance at $570 \mathrm{~nm}$ was recorded to assess the formation of formazan. Inhibitory ratio $(\%)=[1$-absorbance (test)/absorbance (control)] x 100\%, where the test and control correspond to treated and untreated cells, respectively.

Cell cycle and apoptosis analysis. The cell cycle phase was measured by assessing the DNA content via flow cytometry as previously described by Zhong et al (16). Briefly, A375 cells $\left(1 \times 10^{5}\right.$ cells/well) were incubated at $37^{\circ} \mathrm{C}$ with SH at final concentrations of 0 (control), 200 and $400 \mu \mathrm{g} / \mathrm{ml}$ for $48 \mathrm{~h}$. The proportion of cells at different phases was determined using a flow cytometer (Cytomics FC 500 MCL; Beckman Coulter, Inc.). MultiCycle AV software (CXP V2.3 WIN7, C30309; Phoenix Flow Systems, Inc.) was used for analysis. Apoptosis rate was measured by staining with Annexin V-FITC (5 $\mu \mathrm{l})$ and PI (5 $\mu \mathrm{l})$ at $4^{\circ} \mathrm{C}$ for $15 \mathrm{~min}$. Annexin $\mathrm{V}^{-} / \mathrm{PI}^{-}$(lower left quadrant) cells were alive cells, Annexin $\mathrm{V}^{+} / \mathrm{PI}$ (lower right quadrant) cells were early apoptotic cells, Annexin $\mathrm{V}^{+} / \mathrm{PI}^{+}$(upper right quadrant) cells were late apoptotic cells and Annexin $\mathrm{V}^{-} / \mathrm{PI}^{+}$ (upper left quadrant) cells were necrotic cells. The proportion of cells was determined using a flow cytometer (Cytomics FC 500 MCL; Beckman Coulter, Inc.) with MultiCycle AV software (CXP V2.3 WIN7, C30309; Phoenix Flow Systems, Inc.). All experiments were performed in three biological duplicates.

Reverse transcription-quantitative (RT-q)PCR analysis. Total RNA was isolated from A375 cells treated with or without SH $(400 \mu \mathrm{g} / \mathrm{ml})$ for 6 and $48 \mathrm{~h}$ using the Takara MiniBEST universal RNA extraction kit (Takara Bio, Inc.). PrimeScript RT reagent kit with gDNA Eraser (Takara Bio, Inc.) and SYBR ${ }^{\circledR}$ Fast qPCR Mix (Takara Bio, Inc.) were used for the CFX96 real-time PCR system (Bio-Rad Laboratories, Inc.). All operations were performed in accordance with the manufacturer's instructions. The thermocycling conditions consisted of initial denaturation at $95^{\circ} \mathrm{C}$ for $30 \mathrm{sec}$, followed by 40 cycles at $95^{\circ} \mathrm{C}$ for $5 \mathrm{sec}$ and $60^{\circ} \mathrm{C}$ for $30 \mathrm{sec}$. GAPDH was used as an internal reference gene, and gene expression was quantified using the comparative $2^{-\Delta \Delta C q}$ method (18). PCR primer sequences are summarised in Table I.

Western blot analysis. A375 cells treated with or without SH $(400 \mu \mathrm{g} / \mathrm{ml})$ for $48 \mathrm{~h}$ were collected for western blot analysis as previously described (12). Briefly, the total proteins were extracted using the SD-001 buffer (Invent Biotechnologies, Inc.) and supplemented with protein phosphatase inhibitor (Beijing Solarbio Science \& Technology Co., Ltd.), and the protein concentrations were determined using a BCA protein assay kit. A total of $40 \mu \mathrm{g}$ protein/lane was separated via 4-12\% SDS-PAGE and then transferred to a polyvinylidene difluoride membrane. After blocking with 5\% BSA (Merck KGaA) for $1 \mathrm{~h}$ at room temperature, membranes were incubated with the corresponding primary antibodies at $4^{\circ} \mathrm{C}$ overnight. Subsequently, membranes were washed with PBS three times and incubated with the corresponding secondary antibodies at room temperature for $1 \mathrm{~h}$. Primary antibodies $(1: 1,000)$ against p21 (cat. no. ab188224), CyclinD (cat. no. ab28283), CDK2 (cat. no. ab32147), CDK4 (cat. no. ab131469), CDK6 (cat. no. ab131469), Bcl-2 (cat. no. ab32124) and $\beta$-actin (cat. no. ab8226) were obtained from Abcam. The secondary antibody was Alexa Fluor 790-conjugated goat anti-rabbit IgG H\&L (1:10,000; cat. no. ab175781; Abcam). The membranes were washed with PBS three times at room temperature, and the protein signals were visualised using the ChemiDoc touch imaging system (Bio-Rad Laboratories, Inc.) with Image Lab touch software (version 1.2; Chameleon Power).

Immunofluorescence analysis. A375 cells on a slide were fixed in $4 \%$ paraformaldehyde in PBS for $20 \mathrm{~min}$ at room 
Table I. Primers used for reverse transcription-quantitative PCR.

\begin{tabular}{|c|c|c|}
\hline Gene & Forward primer $\left(5^{\prime}-3^{\prime}\right)$ & Reverse primers $\left(5^{\prime}-3^{\prime}\right)$ \\
\hline TGFB & GGACCAGTGGGGAACACTAC & AGAGTCCCTGCATCTCAGAGT \\
\hline Smad2 & CCATACCAAGGTCTCTTGATGGT & ACTGTGAAGATCAGGCCAGC \\
\hline Smad4 & GCAGTCCTACTTCCAGTCCAG & CCCATCTGAGTCTAATGCTACC \\
\hline $\mathrm{p} 21$ & AGCGACCTTCCTCATCCACC & AAGACAACTACTCCCAGCCCCATA \\
\hline p53 & AGCTTTGAGGTGCGTGTTTGTG & TCTCCATCCAGTGGTTTCTTCTTTG \\
\hline p27 & AAAAGCAACAGAAACCTATCCTCAC & ATTCAAAACTCCCAAGCACCTC \\
\hline Cyclin A & TGGAAAGCAAACAGTAAACAGCC & GGGCATCTTCACGCTCTATTT \\
\hline Cyclin B & GCAGCACCTGGCTAAGAATGT & GCCTTGGCTAAATCTTGAACT \\
\hline Cyclin C & GCAGGACATGGGCCAAGAAGA & TATCCACAGAAAGCTCAGCAAACCA \\
\hline Cyclin D & CCCTCGGTGTCCTACTTCAAATGT & GGAAGCGGTCCAGGTAGTTCAT \\
\hline Cyclin E & GCCAGCCTTGGGACAATAATG & CTTGCACGTTGAGTTTGGGT \\
\hline CDK1 & GGATGTGCTTATGCAGGATTCC & CATGTACTGACCAGGAGGGATAG \\
\hline CDK2 & CCAGGAGTTACTTCTATGCCTGA & TTCATCCAGGGGAGGTACAAC \\
\hline CDK3 & CCAGCTCTTTCGTATCTTTCGT & TTCCTGGTCCACTTAGGGAAG \\
\hline CDK4 & ATGGCTACCTCTCGATATGAGC & CATTGGGGACTCTCACACTCT \\
\hline CDK5 & GGAAGGCACCTACGGAACTG & GGCACACCCTCATCATCGT \\
\hline CDK6 & TCTTCATTCACACCGAGTAGTGC & TGAGGTTAGAGCCATCTGGAAA \\
\hline CDK7 & ATGGCTCTGGACGTGAAGTCT & GCGACAATTTGGTTGGTGTTC \\
\hline $\mathrm{Rb}$ & TTGTAACGGGAGTCGGGAGA & CAGCGAGCTGTGGAGGAG \\
\hline E2F1 & CACTTTCGGCCCTTTTGCTC & GTGCTCTCACCGTCCTACAC \\
\hline DP1 & ATGGCTCAGGGAACTGTTGG & GGTCACTGGCAGAGAACCTT \\
\hline Bad & TTGGGGTGAGACCTGTGCG & CTCAGTCTCCССТCAGAACCC \\
\hline Bik & CATTCCAAAGAATCGAAGTCC & AATGTCTGAAGTAAGACCCСТCT \\
\hline $\operatorname{Bax}$ & CCCGAGAGGTCTTTTTCCGAG & CCAGCCCATGATGGTTCTGAT \\
\hline Bid & AGGAAGCCAAACACCAGTAGG & TGTGAACCAGGAGTGAGTCGG \\
\hline Bim & TTTCTTGCGTTTCTCAGTCCG & CGCCACTACCACCACTTGATT \\
\hline Bcl-Xs & TCCCCATGGCAGCAGTAAAG & TCCACAAAAGTATCCTGTTCAAAGC \\
\hline Bak & GCAGGCTGATCCCGTCC & CTGCGGAAAACCTCCTCTGT \\
\hline $\mathrm{Bcl}-2$ & GGTGGGGTCATGTGTGTGG & CGGTTCAGGTACTCAGTCATCC \\
\hline Bcl-xl & GCTGGGACACTTTTGTGGAT & GAGCCCAGCAGAACTACACC \\
\hline Bcl-w & CTTTGTCTTTGGGGCTGCAC & CCATCCACTCCTGCACTTGT \\
\hline Ras & AAGTGTGTGCTCTCCTGACG & CACAAGGGAGGCTGCTGAC \\
\hline Raf & CAACTGATTGCACTGACTGCC & CCTGGAATTGCTCTGGGGTT \\
\hline MEK1 & CTTCGCAGAGCGGCTAGG & CAGCCCGGGATTCCCTTC \\
\hline ERK1 & GAGATGGATGTGGGTTCCAGTC & TTCTCATCGTAGCCACTCACC \\
\hline GAPDH & GGACCTGACCTGCCGTCTAG & GTAGCCCAGGATGCCCTTGA \\
\hline
\end{tabular}

CDK, cyclin-dependent kinase; E2F1, transcription factor E2F1; DP1, transcription factor DP-1.

temperature, and then washed with PBS for 3 times. Cells were blocked with 3\% BSA (Merck KGaA) for 30 min at room temperature, and then primary antibodies against CyclinD (cat. no. ab28283) and Bcl-2 (cat. no. ab32124) (both 1:200; Abcam) were added at $4^{\circ} \mathrm{C}$ overnight. Subsequently, the slides were washed with PBS and incubated with DyLight 488-conjugated goat anti-rabbit IgG (1:200; cat. no. BA1127; Wuhan Boster Biological Technology, Ltd.) for $1 \mathrm{~h}$ at room temperature. The slides were mounted with DAPI-containing medium (C1002; Beyotime Institute of Biotechnology) for counterstaining of nuclei and then observed under a fluorescence microscope (Nikon Eclipse 80i; Nikon Corporation) at x100 magnification.
Statistical analysis. Data were expressed as the mean \pm SD. Statistical analysis was performed using SPSS 16.0 software (SPSS, Inc.). One-way ANOVA was used to analyse statistical differences between groups under different conditions, followed by Tukey's post-hoc test. $\mathrm{P}<0.05$ was considered to indicate a statistically significant difference.

\section{Results}

SH inhibits proliferation of A375 melanoma cells. To examine the effects of SH on melanoma cells, A375 cells were treated with different concentrations of SH $(0,50,100,200$ and $400 \mu \mathrm{g} / \mathrm{ml}$ ) for 24, 36 and $48 \mathrm{~h}$ (Fig. 1). As shown in Fig. 1A 
A

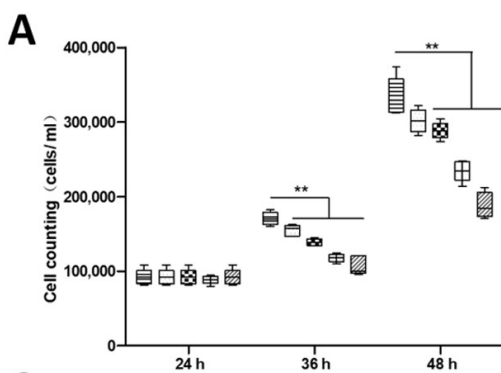

C

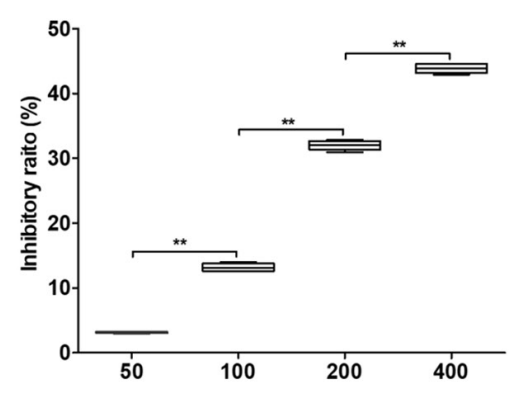

B

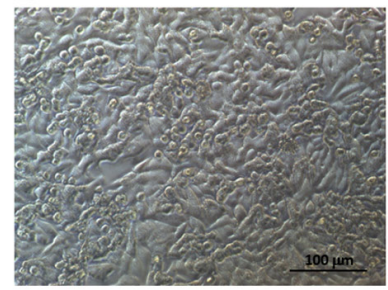

Control

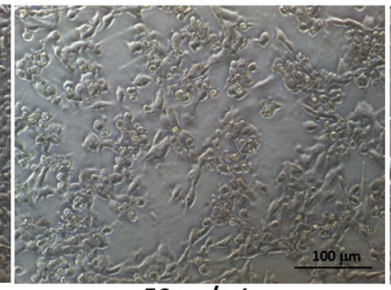

$50 \mu \mathrm{g} / \mathrm{ml}$

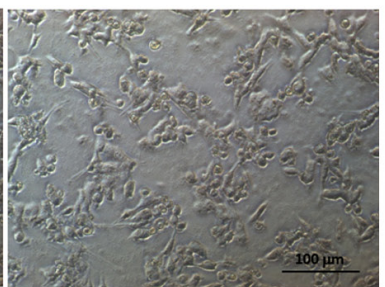

$100 \mu \mathrm{g} / \mathrm{ml}$

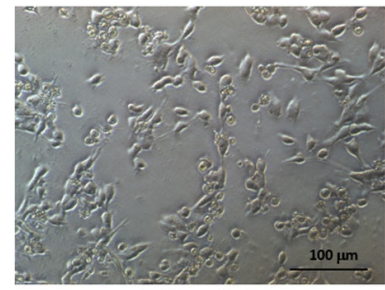

$200 \mu \mathrm{g} / \mathrm{ml}$

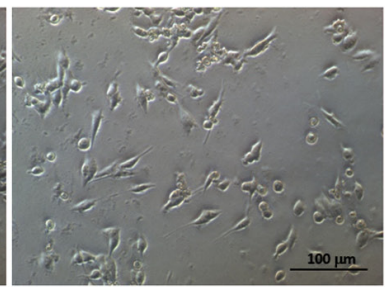

$400 \mu \mathrm{g} / \mathrm{ml}$

Figure 1. Inhibitory effect of SH on A375 cells in vitro. (A) Cell counting of A375 cells under treatment with various concentrations of SH (0-400 $\mu \mathrm{g} / \mathrm{ml})$ at 24,36 and $48 \mathrm{~h}$. (B) Morphological observation of A375 cells under treatment with various concentrations of SH $(0-400 \mu \mathrm{g} / \mathrm{ml})$ at $48 \mathrm{~h}$. Scale bar, $100 \mu \mathrm{m}$. (C) MTT colorimetric analysis of A375 cells under treatment with various concentrations of SH $(0-400 \mu \mathrm{g} / \mathrm{ml})$ at $48 \mathrm{~h}$. Values are presented as the mean $\pm \mathrm{SD}$ ( $\mathrm{n}=5$ each group). ${ }^{* *} \mathrm{P}<0.01$. SH, Sanghuangporus vaninii extract.

and B, treatment of A375 cells with $\mathrm{SH}$ for 36 and 48 h significantly decreased cell viability compared with the control group in a dose-dependent manner. Particularly, $400 \mu \mathrm{g} / \mathrm{ml}$ SH effectively inhibited proliferation of A375 cells, along with a significant inhibitory ratio of $>43 \%$ (Fig. 1B and C). Hence, SH suppressed the proliferation of A375 cells in vitro, suggesting that SH may be used as a cell proliferation inhibitor for the treatment of melanoma.

SH induces cell cycle arrest at the S phase and apoptosis. For further investigations on the inhibitory mechanism of SH on A375 cells, flow cytometry was conducted to assess whether the anti-proliferative effect of $\mathrm{SH}$ on A375 cells was mediated by cell cycle arrest. Under the higher inhibitory concentrations of 200 and $400 \mu \mathrm{g} / \mathrm{ml}$, SH significantly increased the number of cells in S phase (Fig. 2). At $200 \mu \mathrm{g} / \mathrm{ml}$, SH significantly decreased the number of cells at the $G_{2} / M$ phase, while the number of cells at the $G_{0} / G_{1}$ phase significantly decreased with $400 \mu \mathrm{g} / \mathrm{ml} \mathrm{SH}$ (Fig. 2). Hence, SH mainly induced cell cycle arrest of A375 cells at the $\mathrm{S}$ phase.

Flow cytometry with Annexin V-FITC and PI staining was then used to examine the contribution of apoptosis to the SH-mediated inhibition of cell proliferation. SH treatment significantly increased early apoptotic, late apoptotic and necrotic cells compared with the control group (Fig. 3). Hence, $\mathrm{S}$-phase arrest and apoptosis may be mainly involved in the anti-proliferative effect of $\mathrm{SH}$ on A375 cells.

SH induces $S$ phase arrest via p21-cyclin-CDK complex signalling pathways. To further elaborate the molecular mechanism underpinning the anti-proliferative action of $\mathrm{SH}$, RT-qPCR analysis was performed using A375 cells treated with $400 \mu \mathrm{g} / \mathrm{ml} \mathrm{SH}$ for 6 and $48 \mathrm{~h}$, since $400 \mu \mathrm{g} / \mathrm{ml} \mathrm{SH}$ exhibited an increased ability of $\mathrm{S}$ phase arrest. A number of key genes involved in cell cycle were analysed. As shown in Fig. 4A, the expression levels of $p 21$ were significantly upregulated, whereas those of $p 27, C y c l i n s A, C, D$ and $E$, and $C D K 1,2,4,5$ and 6 were significantly downregulated after $48 \mathrm{~h}$ of SH treatment. No significant difference was observed in the expression levels of TGFB, Smad2, Smad4, p53, CyclinB, CDK3, CDK7 and $R b$. Additionally, transcription factors $E 2 F 1$ and $D P 1$ were significantly downregulated after $48 \mathrm{~h}$ of SH treatment.

To confirm the difference in gene expression, western blot and immunofluorescence analyses were conducted. As shown in Fig. 4B, the expression levels of the proteins encoded by some of the aforementioned key genes, such as p21, CyclinD, CDK2, CDK4 and CDK6, were consistent with their gene expression levels after $48 \mathrm{~h}$ of SH treatment. Immunofluorescence analysis of CyclinD-stained cells revealed that the green fluorescence signals in nuclei were strong without SH treatment, while after treatment with $400 \mu \mathrm{g} / \mathrm{ml} \mathrm{SH}$ for $48 \mathrm{~h}$, the number of cells decreased markedly compared with the control, and the green fluorescence signals were weaker (Fig. 4C). Therefore, SH may inhibit A375 cell proliferation by inducing cell cycle arrest at the $\mathrm{S}$ phase by upregulating $\mathrm{p} 21$ expression, which inhibits the expression levels of cyclin-CDK complexes.

SH induces apoptosis by downregulating Bcl-2 expression. Several key genes involved in apoptosis were analysed by RT-qPCR analysis using A375 cells treated with $400 \mu \mathrm{g} / \mathrm{ml} \mathrm{SH}$ for 6 and $48 \mathrm{~h}$, since $400 \mu \mathrm{g} / \mathrm{ml} \mathrm{SH}$ strongly induced apoptosis. The relative mRNA expression levels of pro-apoptotic genes (Bad, Bik, Bid, Bim and Bak) decreased under SH treatment. The expression levels of Bik and Bim decreased at $6 \mathrm{~h}$ significantly, Bid and Bak were downregulated at 48 h significantly, and the relative mRNA expression levels of $\mathrm{Bad}$ decreased at 6 and 48 h significantly (Fig. 5A). No significant difference was observed in the expression levels of MAPK signalling pathway-associated genes (Ras, Raf, MEK1 and ERK1; Fig. 5A). The anti-apoptotic gene $\mathrm{BCl}$-2 was significantly downregulated 


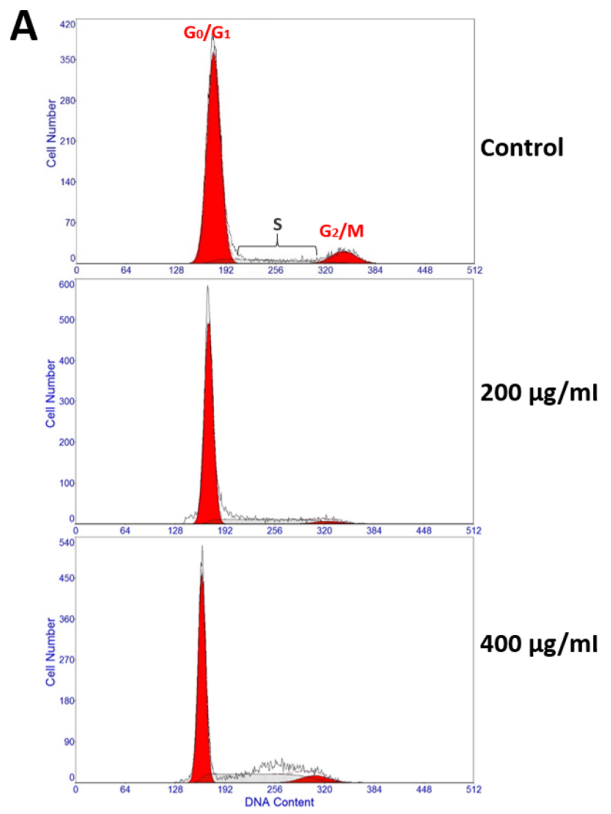

\section{B}

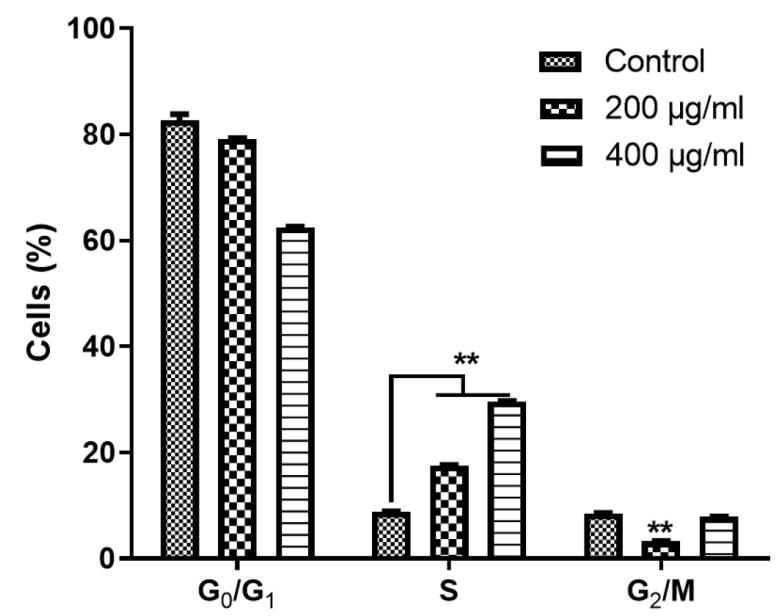

Figure 2. Cell cycle analysis of A375 cells. (A) Cell-cycle distribution was based on 2N and 4N DNA content for DNA content analysis using MultiCycle AV software. (B) Percentage of cells at different cell cycle phases. ${ }^{* *} \mathrm{P}<0.01$ vs. control. The analyses were performed in three duplicates for statistical evaluation. SH, Sanghuangporus vaninii extract.
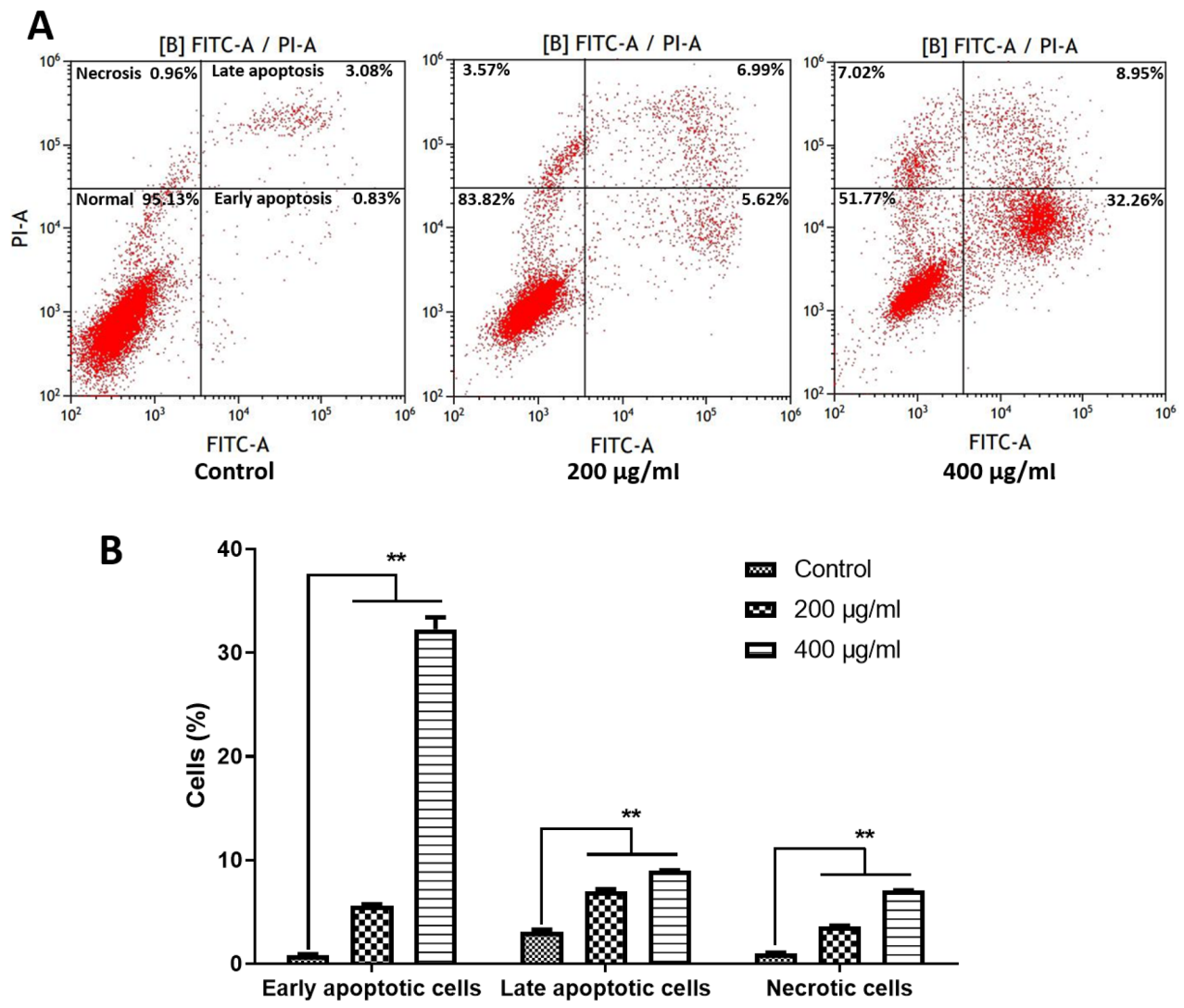

Figure 3. Apoptosis analysis of A375 cells. (A) Different groups of cells were stained with FITC-conjugated Annexin V and PI, and apoptosis was determined by flow cytometry. (B) Percentage of different types of cells. ${ }^{* *} \mathrm{P}<0.01$ vs. control. The analyses were performed in three duplicates for statistical evaluation. $\mathrm{SH}$, Sanghuangporus vaninii extract.

after $\mathrm{SH}$ treatment at $48 \mathrm{~h}$ (Fig. 5A), consistent with the western blot analysis indicating significantly decreased Bcl-2 protein expression (Fig. 5B). Immunofluorescence analysis of $\mathrm{Bcl}-2$-stained cells indicated that the cell density decreased markedly after treatment with $400 \mu \mathrm{g} / \mathrm{ml} \mathrm{SH}$ for $48 \mathrm{~h}$, and the green fluorescence signals were weaker than in the control group (Fig. 5C). The current results suggested that SH may induce apoptosis by downregulating $\mathrm{Bcl}-2$ expression. 


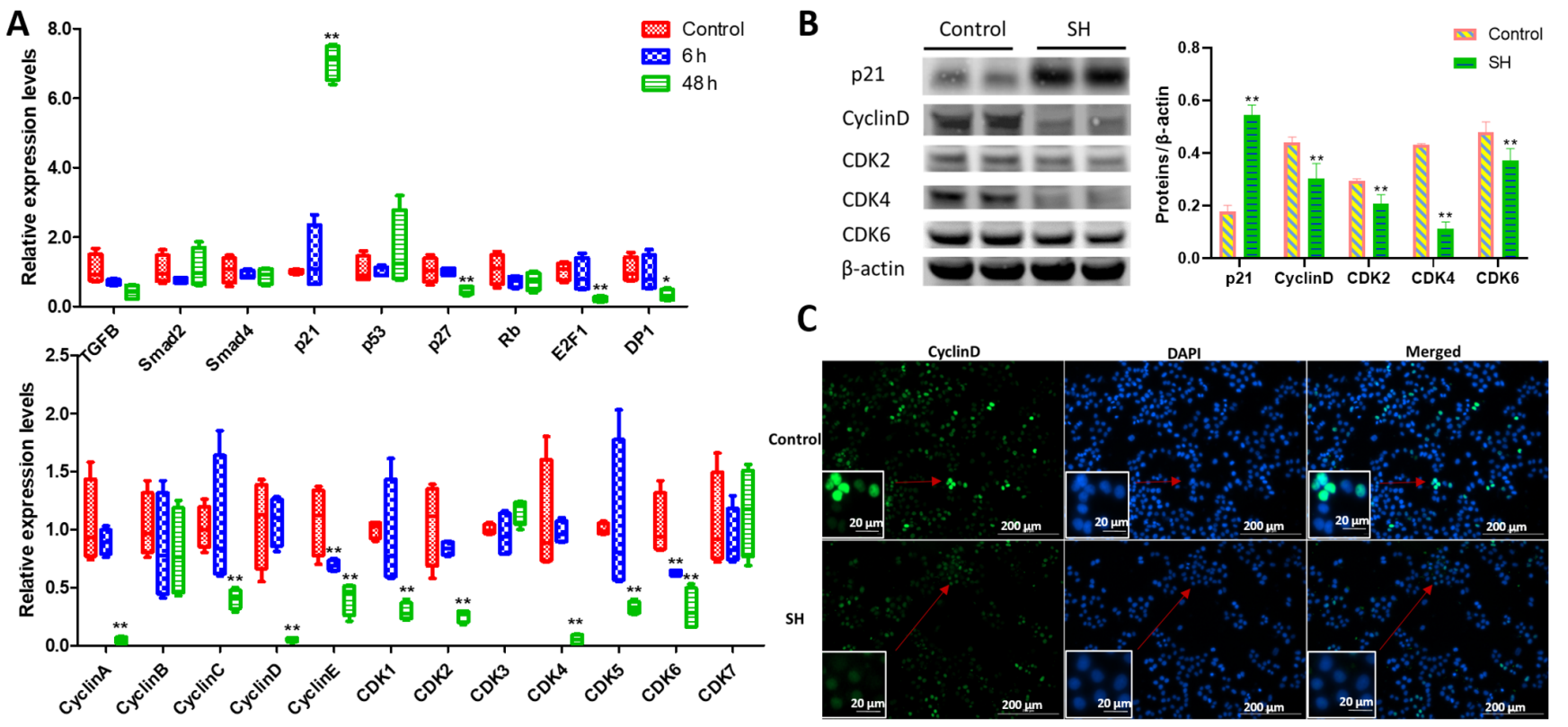

Figure 4. Analysis of cell cycle arrest-associated genes in A375 cells. (A) Reverse transcription-quantitative PCR analysis of cell cycle arrest-associated genes in A375 cells following treatment with SH $(400 \mu \mathrm{g} / \mathrm{ml})$ for 6 and $48 \mathrm{~h}$. (B) Western blot analysis of cell cycle arrest-associated proteins in A375 cells following treatment with SH $(400 \mu \mathrm{g} / \mathrm{ml})$ for $48 \mathrm{~h}$. (C) Immunofluorescence analysis of CyclinD in A375 cells following treatment with SH (400 $\mu \mathrm{g} / \mathrm{ml})$ for $48 \mathrm{~h}$. Data are presented as the mean $\pm \mathrm{SD}$ from four biological duplicates per group. ${ }^{*} \mathrm{P}<0.05$ and ${ }^{* *} \mathrm{P}<0.01$ vs. the control. SH, aqueous extracts of Sanghuangporus vaninii; CDK, cyclin-dependent kinase; E2F1, transcription factor E2F1; DP1, transcription factor DP-1.
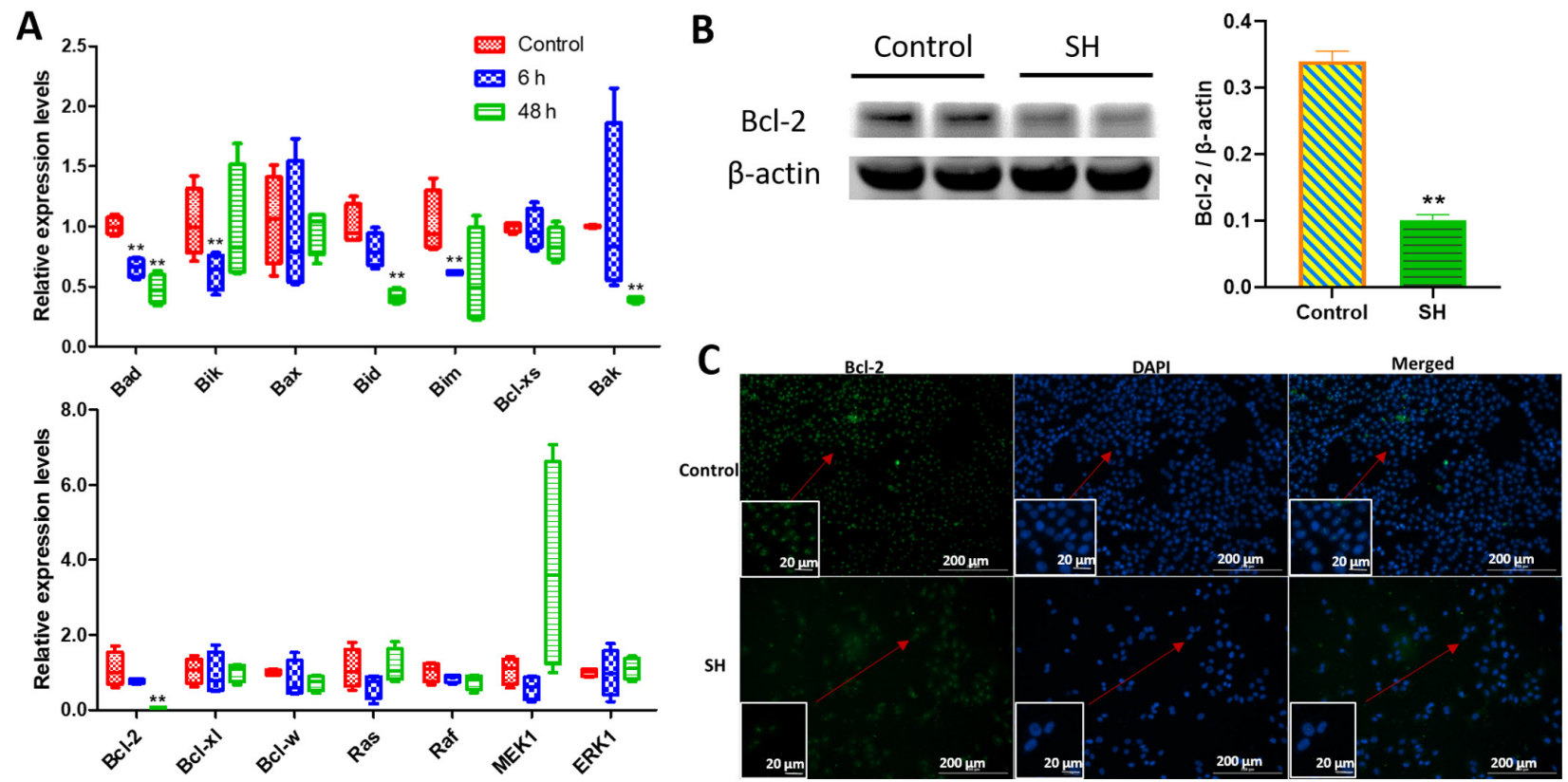

Figure 5. Analysis of apoptosis-associated genes in A375 cells. (A) Reverse transcription-quantitative PCR analysis of apoptosis-associated genes in A375 cells following treatment with SH $(400 \mu \mathrm{g} / \mathrm{ml})$ for 6 and $48 \mathrm{~h}$. (B) Western blot analysis of Bcl-2 expression in A375 cells following treatment with SH (400 $\mu \mathrm{g} / \mathrm{ml})$ for $48 \mathrm{~h}$. (C) Immunofluorescence analysis of Bcl-2 in A375 cells following treatment with SH (400 $\mu \mathrm{g} / \mathrm{ml})$ for $48 \mathrm{~h}$. Data are presented as the mean \pm SD from four biological duplicates per group. ${ }^{* *} \mathrm{P}<0.01$ vs. the control. SH, aqueous extracts of Sanghuangporus vaninii.

\section{Discussion}

Melanoma is one of the most aggressive types of cancer. No specific treatment has been developed to cure patients with metastasis (1). Natural products are important sources for the identification of novel drugs for the treatment of various diseases due to their low toxicity and compelling efficacy (19). Sanghuang mushrooms grow mainly on wild mulberry tree trunks and possess multifunctional bioactivities, such as anti-carcinogenesis, anti-fungal and immunomodulatory activities, as well as anti-diabetic/hyperlipidaemic effects $(5,6)$, presenting Sanghuang as a potential natural product reservoir. Extracts from S. gilvus and S. baumii inhibit murine or human melanoma cell proliferation both in vitro and in vivo $(10,15,16)$. However, to the best of our knowledge, whether $S$. vaninii affects melanoma development has not been revealed. In the present 


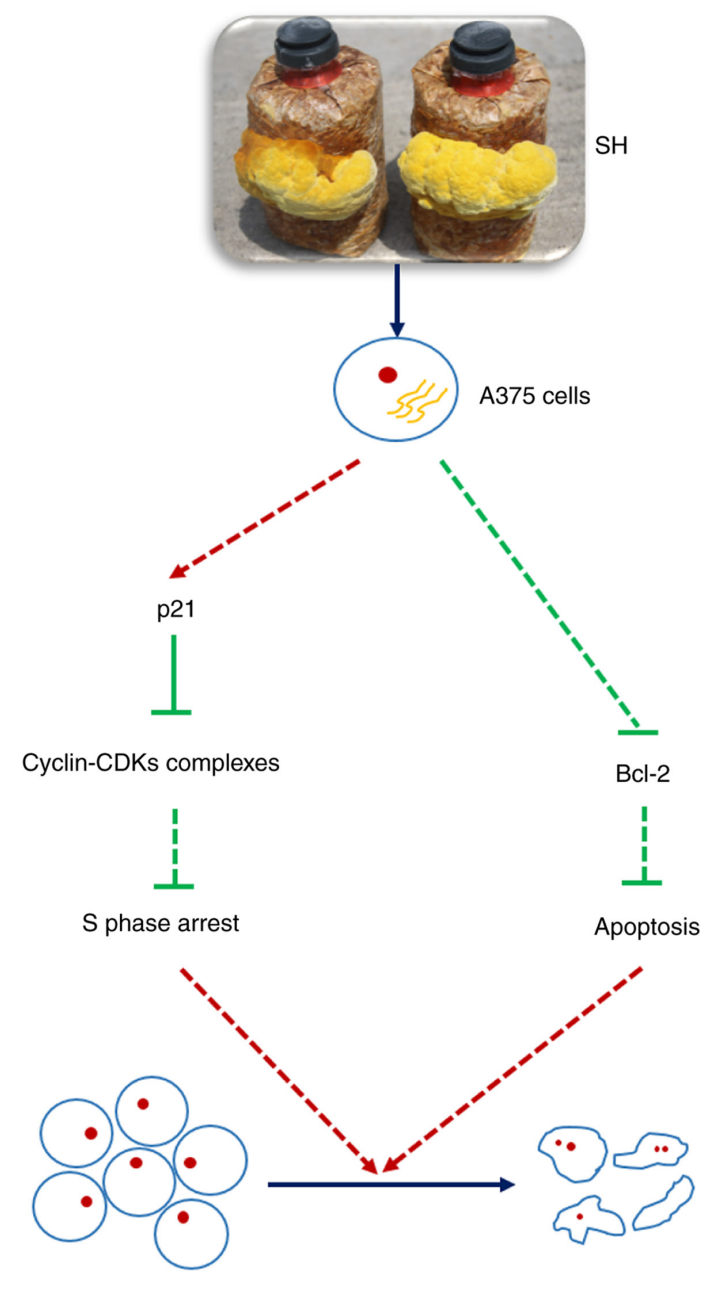

Figure 6. Proposed regulatory mechanism of SH on A375 cells. SH, aqueous extracts of Sanghuangporus vaninii; CDK, cyclin-dependent kinase.

study, it was revealed that aqueous extracts of $S$. vaninii $(\mathrm{SH})$ exerted a proliferation inhibitory effect on human melanoma cells, providing an alternative Sanghuang candidate for developing effective interventions against melanoma.

In general, cancer is well-characterised by continuous cell proliferative ability; hence, interruptions on cancer cell cycle may hinder cancer development (20). In the present study, the effects of SH on melanoma A375 cell proliferation were assessed by MTT assay, revealing that SH exerted a prominent anti-proliferative action in a dose-dependent manner. Perturbations of cell proliferation can be largely attributed to abnormal modulations on cell cycle and apoptosis. Therefore, flow cytometry assays were performed to determine the underlying mechanism. Notably, SH treatment induced cell cycle arrest at the $\mathrm{S}$ phase, as exhibited by the increased cell number at the $S$ phase and decreased cell number at the $G_{0} / G_{1}$ phase, suggesting that $\mathrm{SH}$ may act as a potential proliferation inhibitor of melanoma cells. Consistently with the current results, $S$. baumii can suppress the proliferation of human melanoma A375 cells by promoting cell cycle arrest at the $\mathrm{S}$ phase (15). Moreover, protocatechualdehyde isolated from $S$. gilvus can induce cell cycle arrest in HT29 and B16F10 cells $(12,16)$. Overall, cell cycle arrest may be the general regulatory mechanism of Sanghuang family members in inhibiting the proliferation of cancer cells.
The cell cycle is highly orchestrated by a series of events and signalling pathways, among which cyclins and CDKs are essential in mediating the cell cycle (21). The activity of CDKs is mainly regulated by binding of cyclin proteins/CDK inhibitors and phosphorylation. High levels of p21, also known as CDK inhibitor 1A, can inhibit the functions of CyclinD-CDK4/6, CyclinE-CDK2 and CyclinA-CDK2 complexes, leading to the interruption of the cell cycle $(20,22)$. Moreover, p21 participates in multiple tumour suppressor pathways, including p53-dependent and -independent mechanisms (20,22). p53 is a transcription factor that functions as a well-known tumour suppressor and is involved in both the $G_{0} / G_{1}$ and $G_{2} / M$ cell cycle phases (23). In our previous study, it has been demonstrated that protocatechualdehyde isolated from $S$. givlvus induces S-phase arrest in HT-29 cells via the activation of the CyclinD1/E-CDK2 signalling pathway (12). To investigate the regulatory mechanism of $\mathrm{SH}$ on cell cycle arrest in A375 cells, the expression levels of cell cycle arrest-associated genes were assessed via RT-qPCR, western blot and immunofluorescence analyses. $p 21$ was significantly upregulated, and CyclinD-CDK4/6, CyclinE-CDK2 and CyclinA-CDK2 complexes were downregulated in A375 cells. No significant differences were observed in the relative mRNA expression levels of $p 53$ and upstream genes (TGFB, Smad2 and Smad4) under $\mathrm{SH}$ treatment, suggesting that $\mathrm{SH}$ may promote $\mathrm{p} 21$ expression independently of p53 signalling. The expression levels of $E 2 F 1$ and $D P 1$ were significantly decreased after $48 \mathrm{~h}$ of treatment, and this may be caused by the downregulation of CyclinA and CDK1. Therefore, the current results suggested that $\mathrm{SH}$ treatment in $\mathrm{A} 375$ cells upregulated $p 21$ expression to inhibit Cyclin-CDK complexes, resulting in cell cycle arrest at the $\mathrm{S}$ phase.

Apoptosis is an evolutionarily conserved process that serves an essential role in organism development and tissue homeostasis (24). However, cancer cells lose their ability to undergo apoptosis-induced death, leading to uncontrolled proliferation. It has been previously demonstrated that protocatechualdehyde isolated from S. gilvus induces apoptosis in HT-29 and B16F10 cells $(12,16)$. In the present study, SH induced apoptosis in A375 cells. Under SH treatment, the expression levels of pro-apoptotic genes, such as Bad, Bik, Bid, Bim and Bak, decreased to varying extents, and the anti-apoptotic gene $\mathrm{Bcl}-2$ was significantly inhibited. The $\mathrm{Bcl}-2$ protein family is the key regulator of the intrinsic apoptosis pathway (25). Apoptosis is triggered by pro-apoptotic genes, such as Bim and Bid. This pro-apoptotic chain of events is countered by the anti-apoptotic genes $\mathrm{Bcl}-2, \mathrm{Bcl}-\mathrm{xl}$ and $\mathrm{Bcl}-\mathrm{w}$, which can block apoptosis by binding and sequestering pro-apoptotic proteins (25). Therefore, the significant decrease of Bcl-2 may lead to apoptosis, even though the expression levels of some pro-apoptotic genes were slightly downregulated. These controversial but interesting results require further research.

The current findings suggested that $\mathrm{SH}$ induced S-phase arrest via p21-cyclin-CDKs complex pathways, and induced apoptosis by inhibiting the expression levels of Bcl-2 (Fig. 6). Specific information about the active component(s) in aqueous extracts of $S$. vaninii should be obtained in future studies on isolation, identification and illustration. In addition, the anti-proliferative effects of SH on human melanoma cells in vivo should be further investigated in future studies. 
In conclusion, the present study revealed that the aqueous extracts of $S$. vaninii fruiting body effectively inhibited the proliferation of human melanoma cells by inducing cell cycle arrest at the $\mathrm{S}$ phase, and this arrest may be mediated by the activation of the p21-Cyclin-CDKs complexes signaling pathways. Additionally, SH induced apoptosis of A375 cells via significantly inhibiting the expression levels of the anti-apoptotic gene $\mathrm{Bcl}-2$ (Fig. 6). The current results suggested that $S$. vaninii fruiting body may be used as a source of drug development for the prevention and treatment of melanoma.

\section{Acknowledgements}

Not applicable.

\section{Funding}

The present study was supported financially by Zhejiang Traditional Chinese Medicine Science and Technology Project (grant no. 2020ZZ016), Science and Technology Department of Zhejiang Province (grant nos. 2018C02003 and LGN18C170005), Zhejiang Medical and Health Science and Technology Project (grant no. 2018K Y250) and Key Laboratory of Creative Agriculture, Ministry of Agriculture and Rural Affairs.

\section{Availability of data and materials}

The datasets used and/or analyzed during the current study are available from the corresponding author on reasonable request.

\section{Authors' contributions}

TY, LL and JH contributed to the conception of the study. SZ, YS, HS, WC, JZ and YL performed the experiments. JH and LL revised the paper and gave final approval. TY, LL and JH are responsible for confirming the authenticity of the data. All authors read and approved the final manuscript.

\section{Ethics approval and consent to participate}

Not applicable.

\section{Patient consent for publication}

Not applicable.

\section{Competing interests}

The authors declare that they have no competing interests.

\section{References}

1. Olbryt M: Molecular background of skin melanoma development and progression: Therapeutic implications. Postepy Dermatol Alergol 36: 129-138, 2019.

2. Davis LE, Shalin SC and Tackett AJ: Current state of melanoma diagnosis and treatment. Cancer Biol Ther 20: 1366-1379, 2019.

3. Lee JH, Cho SM, Kim HM, Hong ND and Yoo ID Immunostimulating activity of polysaccharides from mycelia of Phellinus linteus grown under different culture conditions. J Microbiol Biotechnol 7: 2-55, 1997.
4. Chen H, Tian T, Miao H and Zhao YY: Traditional uses, fermentation, phytochemistry and pharmacology of Phellinus linteus: A review. Fitoterapia 113: 6-26, 2016

5. Chen W, Tan H, Liu Q, Zheng X, Zhang H, Liu Y and Xu L: A review: The bioactivities and pharmacological applications of Phellinus linteus. Molecules 24: 1888, 2019.

6. Huo J, Zhong S, Du X, Cao Y, Wang W, Sun Y, Tian Y, Zhu J, Chen J, Xuan L, et al: Whole-genome sequence of Phellinus gilvus (mulberry Sanghuang) reveals its unique medicinal values. J Adv Res 24: 325-335, 2020.

7. Meera CR,Janardhanan KK andKarunagaran D: Antiproliferative and apoptotic activities of the medicinal mushroom Phellinus rimosus (Agaricomycetes) on HCT116 human colorectal carcinoma cells. Int J Med Mushrooms 20: 935-945, 2018.

8. Wang FF, Shi C, Yang Y, Fang Y, Sheng L and Li N: Medicinal mushroom Phellinus igniarius induced cell apoptosis in gastric cancer SGC-7901 through a mitochondria-dependent pathway. Biomed Pharmacother 102: 18-25, 2018.

9. Im KH, Baek SA, Choi J and Lee TS: Antioxidant, anti-melanogenic and anti-wrinkle effects of Phellinus vaninii. Mycobiology 47: 494-505, 2019.

10. Bae JS, Jang KH, Yim H and Jin HK: Polysaccharides isolated from Phellinus gilvus inhibit melanoma growth in mice. Cancer Lett 218: 43-52, 2005.

11. Bae JS, Jang KH, Yim H, Park SC and Jin HK: Inhibitory effects of polysaccharides isolated from Phellinus gilvus on benzo(a) pyrene-induced forestomach carcinogenesis in mice. World J Gastroenterol 11: 577-579, 2005.

12. Zhong S, Li YG, Ji DF, Lin TB and Lv ZQ: Protocatechualdehyde induces $\mathrm{S}$-phase arrest and apoptosis by stimulating the p27KIP1-Cyclin A/D1-CDK2 and mitochondrial apoptotic pathways in HT-29 Cells. Molecules 21: 934, 2016.

13. Chao W, Deng JS, Li PY, Liang YC and Huang GJ: 3,4-Dihydroxybenzalactone suppresses human non-small cell lung carcinoma cells metastasis via suppression of epithelial to mesenchymal transition, ROS-mediated PI3K/AKT/MAPK/ MMP and NFKB signaling pathways. Molecules 22: 537, 2017.

14. Liu YJ, Lyu JL, Kuo YH, Chiu CY, Wen KC and Chiang HM: The anti-melanogenesis effect of 3,4-dihydroxybenzalacetone through downregulation of melanosome maturation and transportation in B16F10 and human epidermal melanocytes. Int J Mol Sci 22: 2823, 2021.

15. Yang Y, Zhang L, Chen Q, Lu WL and Li N: Antitumor effects of extract of the oak bracket medicinal mushroom, Phellinus baumii (Agaricomycetes), on human melanoma cells A375 in vitro and in vivo. Int J Med Mushrooms 22: 197-209, 2020.

16. Zhong S, Jin Q, Yu T, Zhu J and Li Y: Phellinus gilvus derived protocatechualdehyde induces G0/G1 phase arrest and apoptosis in murine B16 F10 cells. Mol Med Rep 21: 1107-1114, 2020.

17. Huo J, Sun Y, Zhong S, Li Y, Yang R, Xia L, Wang J, Zhang M and Zhu J: Safety evaluation of aqueous extracts of Sanghuangporus vaninii fruiting body in Sprague-Dawley rats. Food Sci Nutr 8: 5107-5113, 2020.

18. Schmittgen TD and Livak KJ: Analyzing real-time PCR data by the comparative C(T) method. Nat Protoc 3: 1101-1108, 2008.

19. Harvey AL, Edrada-Ebel R and Quinn RJ: The re-emergence of natural products for drug discovery in the genomics era. Nat Rev Drug Discov 14: 111-129, 2015.

20. Moussa RS, Park KC, Kovacevic Z and Richardson DR: Ironing out the role of the cyclin-dependent kinase inhibitor, p21 in cancer: Novel iron chelating agents to target p21 expression and activity. Free Radic Biol Med 133: 276-294, 2019.

21. Diaz-Moralli S, Tarrado-Castellarnau M, Miranda A and Cascante M: Targeting cell cycle regulation in cancer therapy. Pharmacol Ther 138: 255-271, 2013.

22. Al Bitar S and Gali-Muhtasib H: The role of the cyclin dependent kinase inhibitor p21cip1/waf1 in targeting cancer: Molecular mechanisms and novel therapeutics. Cancers (Basel) 11: E1475, 2019.

23. Golias $\mathrm{CH}$, Charalabopoulos A and Charalabopoulos K: Cell proliferation and cell cycle control: A mini review. Int J Clin Pract 58: 1134-1141, 2004.

24. Du Toit A: Cell death: Balance through a bivalent regulator. Nat Rev Mol Cell Biol 14: 546-547, 2013.

25. Singh R, Letai A and Sarosiek K: Regulation of apoptosis in health and disease: The balancing act of BCL-2 family proteins. Nat Rev Mol Cell Biol 20: 175-193, 2019. 